\title{
ELECTROMIGRATION EARLY RESISTANCE INCREASE MEASUREMENTS
}

\author{
J. NIEHOF \\ MESA Research Institute, University of Twente, P.O. Box 217, 7500 AE Enschede, The Netherlands \\ AND \\ P. A. FLINN AND T. J. MALONEY \\ Intel Corporation, 2250 Mission College Blvd., SC9-06, Santa Clara, CA 95052, U.S.A.
}

\begin{abstract}
SUMMARY
An early resistance change measurement set-up, using an $\mathrm{AC}$ bridge technique, has been developed, and measurements have been performed. Large sample-to-sample variations occur. The characteristic time for the resistance change curve is shorter for resistance increase (under current stress) than for resistance decay (during recovery).
\end{abstract}

KEY WORDS Electromigration AC bridge Resistance change Void Healing Characteristic time

\section{INTRODUCTION}

Although a lot of work has been performed on electromigration, it is still not at present well understood, and a more detailed understanding of the electromigration process is desired. The most accepted method for electromigration testing has been conventional life-testing of samples until a particular failure condition is reached. Although this method gives information such as median time to failure and activation energies, it does not tell much about a particular subpopulation of the failures which is particularly interesting, the early failure distribution.

\section{THE ERCM}

The momentum exchange between the electrons and the conductor atoms results in an atomic flux in the direction of the electron flow. Owing to this redistribution of atoms void areas are created. We believe that there are three stages in the electromigration failure process: void formation, void movement and void capture, in that order.

In order to study the first stage, void formation, an early resistance change measurement (ERCM) set-up was developed; ${ }^{1}$ this will be described in detail elsewhere. The ERCM uses an alternative electromigration testing method developed by Lloyd and Koch, ${ }^{2}$ to avoid the need to cool the sample to room temperature for measurement when a DC technique $^{3}$ is used. A block diagram of the ERCM set-up is given in Figure 1. The set-up uses an AC Wheatstone bridge technique; this is to measure solely the effect of electromigration. This bridge network compares the resistance of the conductor stripe undergoing high current density DC stressing to an unstressed monitor stripe. The bridge is arranged so that the measured voltage reflects the difference in resistance between the monitor and the stressed stripe only. Sample temperature changes, which are assumed to be the same in both stripes, will therefore be cancelled out of the bridge voltage. A major advantage of the bridge technique over non-bridge techniques ${ }^{4}$ is that no sample temperature drift corrections are required in order to perform accurate resistance change measurements.

Our set-up, as compared with Lloyd's, allows us to perform measurements continuously (no switching is needed). Also no start-up bridge adjustments are required, since this system uses a 16-bit $\mathrm{AD}$ converter giving us enough dynamic range to handle both bridge imbalances caused by initial differences between monitor and stress stripe, and bridge imbalances due to the joule heating in the stress stripe. Instead of using a separate lock-in amplifier we use small and inexpensive on-chip lock-in amplifiers, giving us the possibility to easily expand the set-up to run more samples in parallel.

The ERCM system is controlled by an Intel Multibus computer (using a 386 microprocessor).

\section{EXPERIMENTS}

The ERCM measurements were performed on structures as shown in Figure 2. This is an $\mathrm{Al} / \mathrm{Si}$ metallization structure passivated with $0.7 \mu \mathrm{m}$ thick $\mathrm{SiO}_{2}$.

The stress stripe is placed close to the monitor stripe in order to reduce any Joule heating effects as much as possible. Typical current densities of $2 \mathrm{MA} / \mathrm{cm}^{2}$ and temperatures of $200^{\circ} \mathrm{C}$ were used.

A typical measurement is to observe the resistance changes when stressing the stripe first with a positive current, then reversing the current, and finally with no current. 


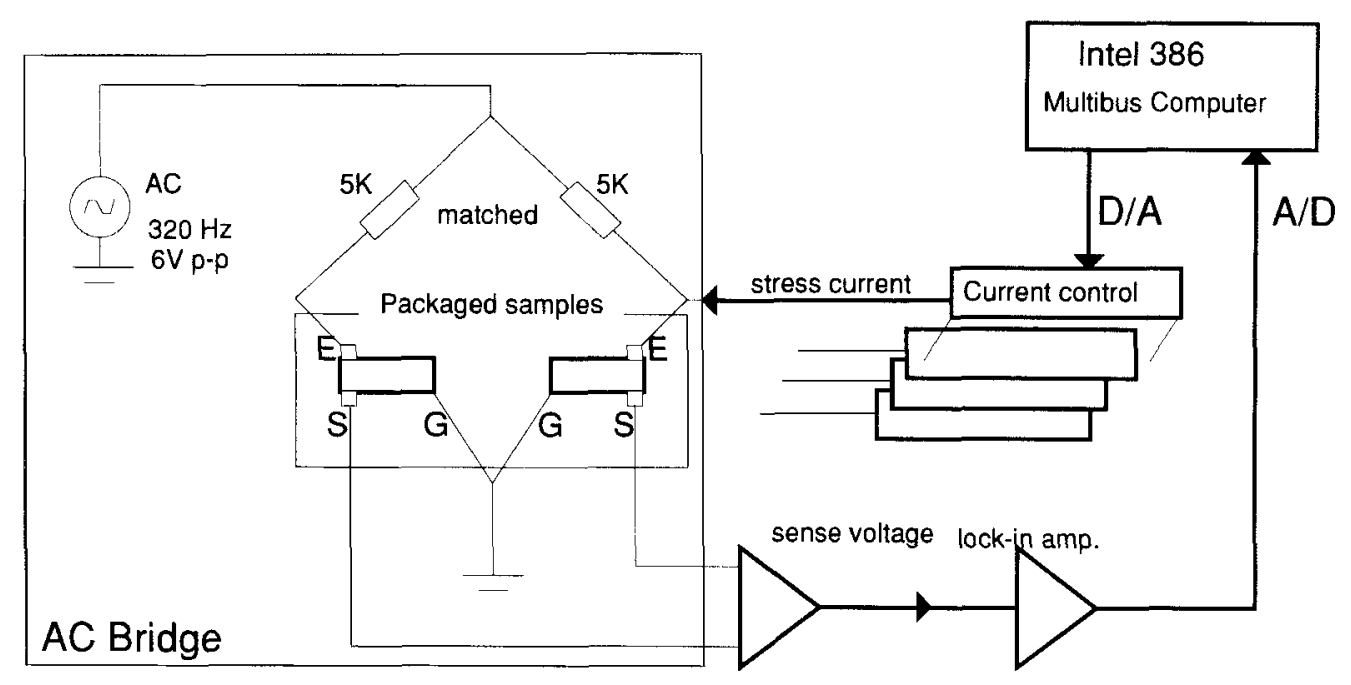

Figure 1. Block diagram of the ERCM

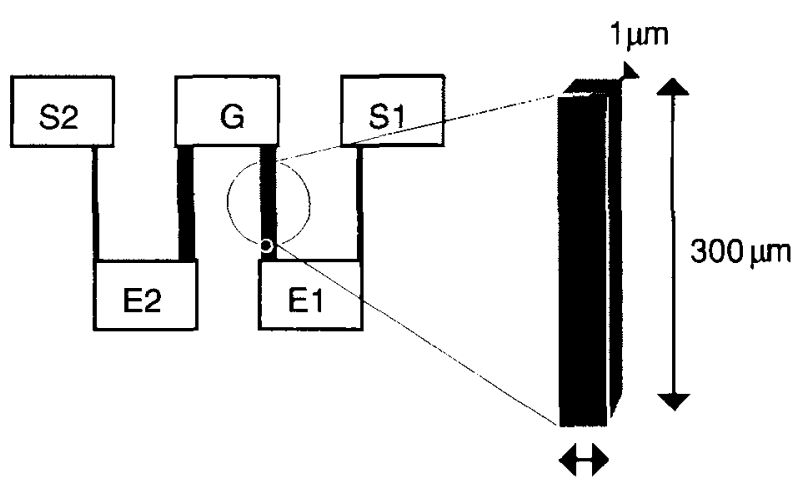

$3 \mu \mathrm{m}$

Figure 2. $\mathrm{ERCM}$ test structure: $\mathrm{S}=$ sense $; \mathrm{E}=$ excitate $\mathrm{G}=$ ground

\section{RESULTS}

During measurements in all cases large sample-tosample variations were observed. Plots showing typical resistance changes for two different conditions are given in Figures 3 and 4. When the stress current is switched on, the resistance starts increasing, initially at an exponential rate, and for longer times at a linear rate. When the current stress is switched off, an exponential resistance decay is observed.

\section{DISCUSSION}

From the resistance change curve in Figure 3, we deduce that as soon as stress is applied to the sample, microvoids caused by supersaturation of vacancies rapidly start to form, giving rise to the exponential increase in resistance. The linear part of the curve results from the growing of these microvoids. The resistance change after current-on can be written as

$$
\Delta R_{\mathrm{on}}(t)=C_{\mathrm{on}}\left(1-\mathrm{e}^{-t / \tau_{\mathrm{incr}}}\right)+L_{\mathrm{incr}} t
$$

where $C_{\text {on }}$ is a constant and $\tau_{\text {incr }}$ is the characteristic time of the exponential resistance increase. $L_{\text {incr }}$ is the linear resistance increase rate.
Resistance recovery, a healing process, is observed (Figure 4) when the current stress is turned off. When the stress is turned off, those microvoids which are unstable will evaporate, and the resistance recovers. We have never observed a total resistance recovery, indicating that some of the microvoids have stabilized into stable, permanent voids, resulting in permanent resistance change $\left(R_{\text {perm }}\right)$. The resistance change after current-off can then be expressed as

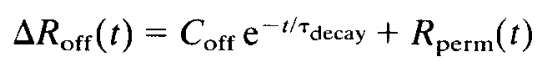

The permanent resistance change $\left(R_{\text {perm }}\right)$ is a function of time, for longer times more microvoids will have stabilized resulting in a larger $R_{\mathrm{perm}}$. Furthermore we believe that $R_{\text {perm }}$ is a function of inhomogeneities/disturbances in the metallization which cause the unstable microvoids to stabilize into permanent voids.

When inspecting the characteristic times $(\tau)$ for the exponential resistance change curves, it shows a larger $\tau$ for the resistance decay than for the resistance increase. The $\tau$ for decay is in good agreement with that found by Lloyd, ${ }^{2}$ typically $3-10 \mathrm{~h}$. Lloyd did not report the exponential increase in resistance, for which we typically found a $\tau$ of $1-3 \mathrm{~h}$. These results disagree with the work of Hinode, ${ }^{4}$ reporting $\tau_{\text {incr }}$ and $\tau_{\text {decay }}$ in the same range having a value of 4 to $16 \mathrm{~min}$.

When comparing the time scales, the time scale for resistance decay indicates that we are not dealing with isolated vacancies. We are dealing with microvoids, not detectable by SEM observations. ${ }^{5,6}$ The complex effects occurring at current reversal are currently under investigation.

\section{CONCLUSIONS AND FUTURE STEPS}

Since the ERCM method is relatively non-destructive (resistance changes are of the order of parts 


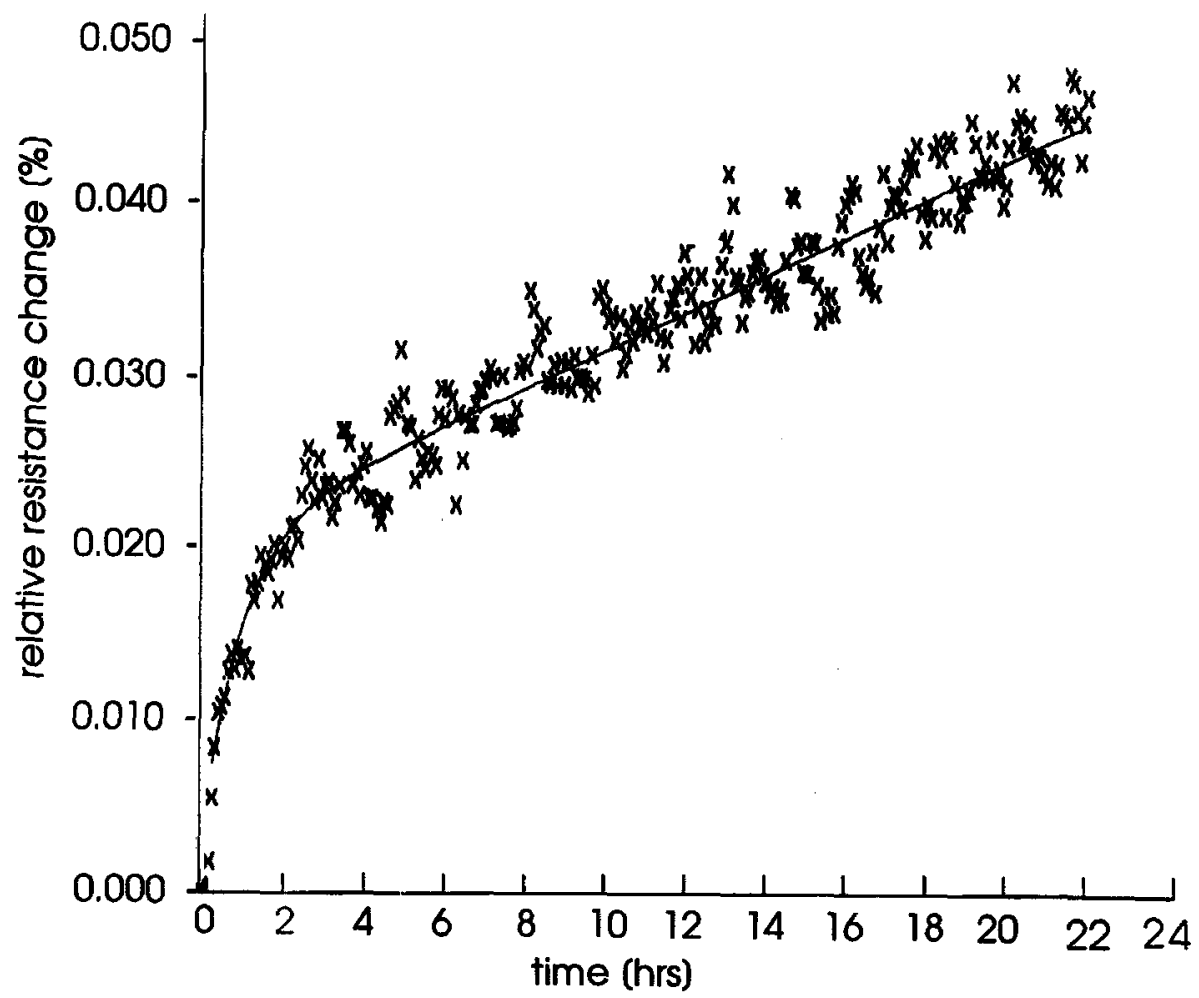

Figure 3. Relative resistance change under current stress

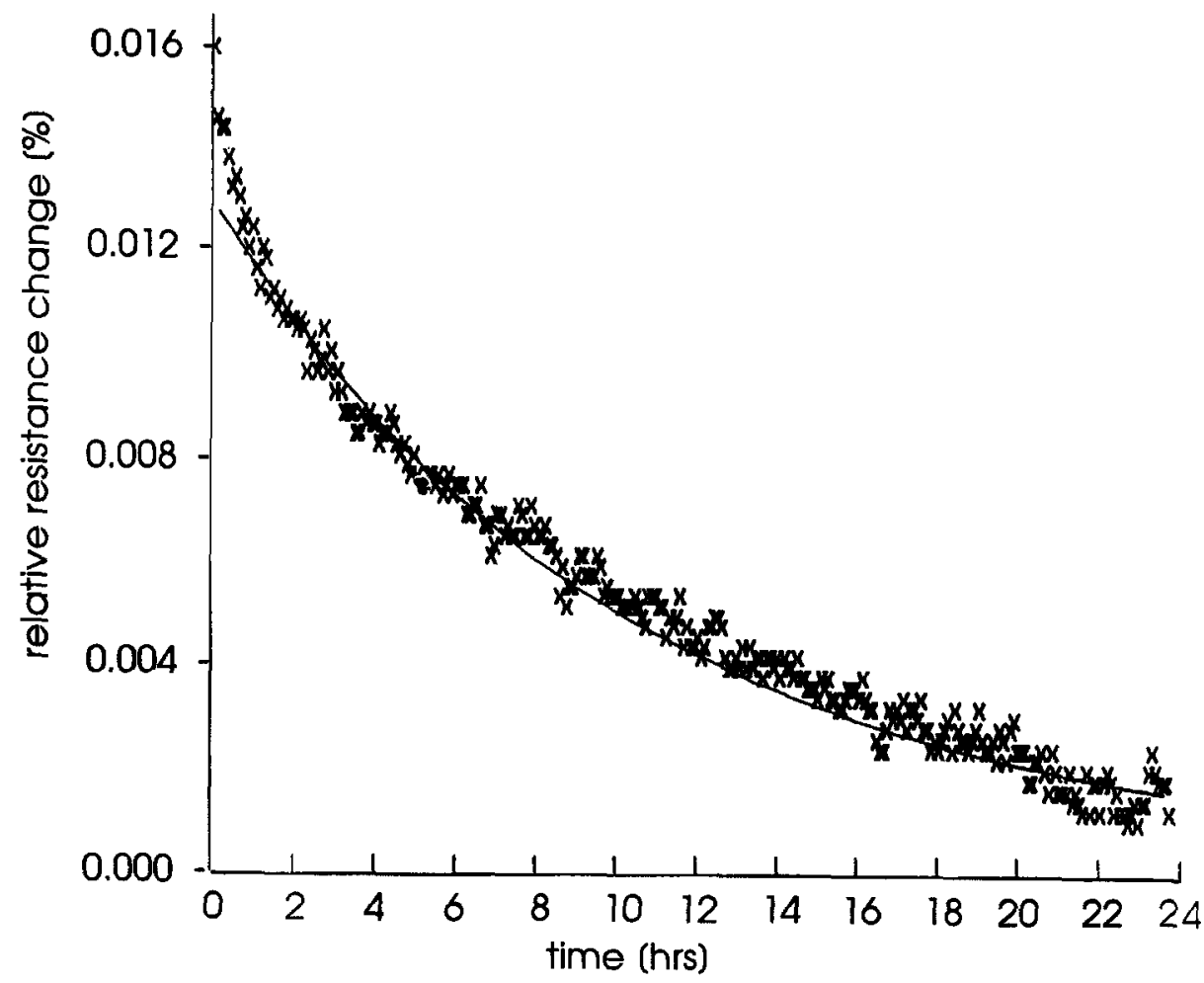

Figure 4. Relative resistance change after removal of current stress

per thousand for a typical test), it is possible to carry out conventional life-testing on samples after an ERCM test.

At the moment we are studying identical strips both with ERCM measurements and with HVSEM. ${ }^{6}$ We believe that when using the samplespecific information from the ERCM and HV-SEM observations it should be possible to gain more insight in the complex phenomena of electromigration.

\section{REFERENCES}

1. P. A. Flinn, T. J. Maloney, M. Eisele, S. T.Sng, B. Patel, V. Aleksiev, E. Abratowski, C. Xie, A. Lee and J. Nichof, Private Communication.

2. J. R. Lloyd, R. H. Koch, 'Study of electromigration-induced 
resistance and resistance increase in $\mathrm{Al}$ thin film conductors', Proc. IRPS 1987, p.161.

3. J. A. Maiz and I. Segura, 'A resistance change methodology for the study of electromigration in Al-Si interconnects', Proc. IRPS, 1988, pp.209-215.

4. K. Hinode, T. Furusawa and Y. Homma, 'Relaxation phenomenon during electromigration under pulsed current', Proc. IRPS, 1992, p.205.

5. E. Castaño, J. Maiz, P. Flinn and M. Madden, 'In situ observations of $\mathrm{dc}$ and ac electromigration in passivated Al lines', Appl. Phys. Lett., 59,(1), (1991) 129-131.

6. M.Madden, T. Marieb, E. Abratowski and P. A. Flinn, 'High resolution observation of void motion in passivated metal lines under electromigration stress' Mat. Res. Soc. Symp. Proc., 265, 33 (1992).

\section{Authors' biographies}

Jan Niehof was born in Boskoop, The Netherlands in 1967. He received his M.Sc. degree in electrical engineering from the University of Twente, The Netherlands in 1990. He is currently working towards his Ph.D. degree in electrical engineering on electromigration in thin films at the University of Twente. His main topic is the modelling of electromigration, concentrating on early resistance changes. He received the Best Poster Award for his contribution to the ESREF symposium in 1992.

Paul A. Flinn received his AB in 1948 and his MS in Physics in 1949 from Columbia University, and his ScD in Metallurgy from MIT in 1952. He taught physics at Wayne University for two years, then joined the staff of the Westinghouse Research Laboratory. In 1963 he joined the faculty of Carnegie Tech (now Carnegie-Mellon University) with a joint appointment in Physics and Metallurgy. In 1971 he received the Argonne University Association Distinguished Appointment at the Argonne National
Laboratory. He has been a Visiting Profesor at the University of Nancy, France and at the Universidade Federal do Rio Grande du Sol, Brasil. He joined Intel in 1978 as a Senior Staff Scientist. In 1984 he was selected for the Intel Researcher in Residence program, and spent the year at Stanford University as a Visiting Professor, investigating the mechanical properties of thin films. In 1985 he was appointed a Consulting Professor in the Materials Science and Engineering department at Stanford. His current interests include the properties of metal and dielectric films used in multilevel interconnections, and fundamental aspects of electromigration. He is a member of Phi Beta Kappa, Tau Beta Pi, Sigma Xi, AIME, AAAS, and a fellow of the American Physical Society.

Timothy J. Maloney received an S.B. degree in physics from the Massachusetts Institute of Technology in 1971, an M.S. in physics from Cornell University in 1973, and a Ph.D. in electrical engineering from Cornell in 1976, where he was a National Science Foundation Fellow. He was a Postdoctoral Associate at Cornell until 1977, when he joined the Central Research Laboratory of Varian Associates, Palo Alto, CA. At Varian until 1984, he worked on III-V semiconductor photocathodes, solar cells and microwave devices, as well as silicon molecular beam epitaxy and MOS process technology. Since 1984 he has been with Intel Corp., Santa Clara, CA, where he has been concerned with integrated circuit ESD protection, CMOS latchup testing, fab process reliability and other component reliability issues. He is now a Senior Staff Engineer at Intel. Dr. Maloney received Best Paper Awards for his contributions to the EOS/ESD Symposium in 1986 and 1990, and was General Chairman for the 1992 EOS/ESD Symposium. He has taught short courses at UCLA, University of Wisconsin, and UC Berkeley. He is also a member of the Electrochemical Society, and a Senior Member of the IEEE. 